\title{
ESTUDIO DE LAS COMUNIDADES DE INTERES PASCICOLA EN UN PUERTO DE MONTAÑA: II EVOLUCION EN LA INTENSIDAD DE SELECCION DEL PASTO POR DOS RAZAS OVINAS (CHURRA Y MERINA)
}

\author{
P.R. Revesado (1), J.S. González (2), P. Frutos (1), G. Ramos (1), I. Alonso
} (1), A. García (1) y F.F. Bermúdez (1).

(1) Estación Agricola Experimental de León, CSIC, Apdo. 788, 24080 León.

(2) Departamento de Producción Animal, Universidad de León, 24071 León.

RESUMEN.-Con objeto de conocer la intensidad de selección del pasto en el ganado ovino y las diferencias entre los animales de raza Churra y de raza Merina en este parámetro, se llevó a cabo un experimento sobre un pasto de "Nardus" en la zona de montaña del Puerto de San Isidro en la provincia de León. Se utilizaron 4 machos adultos castados de cada raza, provistos de fístulas esofágicas para la toma de muestras de la dieta seleccionada por los animales (extrusa). El muestreo tuvo lugar en la Liltima semana de los meses de junio, julio, agosto y septiembre de 1990. En cada muestra se separaron las hojas y los tallos, expresándolos posteriormente como porcentaje de la materia seca. No se encontraron diferencias estadisticamente significativas $(P>0,05)$ entre ambas razas, en la proporción de hojas en las extrusas (88,3 vs $85,3 \%$ para las razas Churra y Merina respectivamente). EI efecto del periodo fue estadisticamente significativo $(P>0,01)$ para la proporción de hojas, con un menor valor $(78 \%)$ en julio. Las diferencias entre periodos, tampoco fueron estadísticamente significativas $(P>0,05)$ en la selección de hojas en la raza Churra, no obstante en la raza Merina se obtuvo un menor valor en la proporción de hojas en las extrusas en el segundo periodo. El indice de selección (\% de hojas en la extrusa $/ \%$ hojas en el pasto) presentó diferencias en los valores correspondientes a los distintos periodos. En la raza Merina durante los meses de junio y julio el valor del indice de selección fue significativamente menor que en los meses de agosto y septiembre, no encontrando diferencias significativas en estos pares de valores. Para la raza Churra, el valor del indice de selección obtenido en el mes de junio fue significativamente menor que el encontrado en los otros tres periodos, entre los cuales no se pudo comprobar la existencia de diferencias estadisticamente significativas. 
ABSTRACT.- In order to know how the grazing period during summer affects to the degree of food selection in mountain areas and consistency of this differences in Churro and Merino genotypes an experiment on "Nardus" grass was carried out. Four oesophageal fistulated wether lambs of each breed were used during the last week of June, July, August and September 1990 and the leaf and steam were separated in each sample. There were no differences $(P>0,05)$ between breed in the proportion of extrusa leaves $(88,3$ and $85,3 \%$ for Churra and Merino respectively). The effect of period was significatively different $(P<0,01)$ for the proportion of leaf, with a lower value $(78 \%)$ in the second period (driest state of grass). There were no differences $(p<0,01)$ in selection index (\% ofleaves in the extrusa /\% of leaves in the grass) among periods. For the Merino genotype the selection index was lower $(P<0,01)$ during June and July periods than august and September values. For the Churra breed the value of index of food selection at June period was lower $(P<0,01)$ than the other three periods.

RÉSUMÉ.-Pour connaître l'intensité de sélection du pâturage chez les ovins et les differences entre les animaux de race Churra et de race Merina concernat ce paramètre, nous avons mis au point une expérience sur un pâturage de "Nardus" dans la zone de montagne du "Port du San Isidro" dans la province de León. Nous avons utilisé 4 mâles adultes castrés de chaque race, pourvus de fistules oesophagiennes pour avoir les échantillons de la diète sélectionnée par les animaux (extrusa). L'expérimentation a eu lieu pendant la dernière semaine des mois de juin, juillet, aôut et septembre 1990. Pour chaque échantillon nous avons séparé les feuilles et les tiges, en le traduisant netérieurement en pourcentage de la matiere sèche. Nous n'avons pas trouvé de différences statistiquement significatives $(P>0,05)$ entre les deux races en ce quiconcerne la proportion de feuilles dans les extrusas $(88,3$ vs $85,3 \%$ pour les races Churra et Merina respectivement). L'effet de la période a été statistiquement significatif $(P<0,01)$ pour le pourcentage de feuilles, avec une valeur inférieure $(78 \%)$ en juillet. Les différences entre les périodes n'ont pas été non plus statistiquement significatives $(P>0,05)$ en ce qui concerne la sélection de feuilles chez la race Churra, cependant chez la race Merina nous avons obtenu une valeur inférieure dans la proportion de feuilles dans les extrusas pendant la seconde période. L'indice de sélection (\% de feuilles dans l'extrusa /\% de feuilles dans le pâturage) a présenté des differences dans les valeurs correspondantes aux differentes périodes. Chez la race Merina pendant les mois de juin et juillet la valeur de l'indice de sélection a été significativement inférieure à celle des mois d'aôutet septembre, sans trouver de différences significatives dans ces deux valeurs. Pour la race Churra, la valeur de l'indice de sélection obtenue pendant le mois de juin a été significativement inférieure à celle trouvée pendant les trois autre périodes, entre les quelles nous n'avons pas pu vérifier l'existence de différences statistiquement significatives.

Key words: Sheep grazing, Intensity offood selection, Evolution, Genotypes differences.

\section{Introducción}

Al tratar de estudiar los sistemas de producción ovina, la primera sorpresa es la reorganización a la que están siendo sometidos sus objetivos; así, frente al interés fundamental de conseguir las máximas producciones a cualquier coste siempre que el precio de venta compensara, en la actualidad 
el interés está ligado a la mejora y conservación del medio natural, manteniendo una población mínima sin cuya condición la degradación medioambiental está asegurada (Hodgson, 1989).

En zonas de montaña, la cubierta vegetal se caracteriza por su dinamismo, por lo que para mantener el paisaje es necesaria la acción especial de los animales sobre la misma (Grant \& Hodgson, 1986). Otra característica de los ecosistemas pastorales de montaña es la variación estacional en la calidad y cantidad de la vegetación, como consecuencia de las variaciones climáticas a lo largo del año.

Debido a las características climáticas y orográficas, la utilización de los pastos de montaña queda limitada a los meses de verano, desde finales de junio a mediados de octubre y aún en este corto periodo de tiempo existe una disminución en la oferta forrajera, tanto cualitativa como cuantitativa, debida a la sequia del verano.

En condiciones de pastos permanentes de montaña, por la diversidad botánica existente, la ingestión depende de manera fundamental de la intensidad de selección que los animales pueden ejercer sobre el pasto en oferta, pero otros condicionantes como es el tiempo de pastoreo diario deben ser tenidos en cuenta, por la relación existente entre la intensidad de selección sobre el pasto, el ritmo de ingestión y el tamaño de cada bocado (Armstrong \& Hodgson, 1986; Forbes, 1988; Illius, 1986).

Las diferencias existentes entre las distintas especies de rumiantes en cuanto a su capacidad para seleccionar diferentes partes de la cubierta vegetal, como consecuencia de su adaptación evolutiva a los diferentes tipos de forraje disponible, han sido puestas de manifiesto por numerosos autores (Belovsky, 1986; Gordon \& lason, 1989; Malacheck \& Balph, 1987; Weston \& Poppi, 1987). Sin embargo, no ha sido suficientemente estudiada la posibilidad de extrapolar estas diferencias interespecíficas a diferentes genotipos, teniendo en cuenta que la evolución y adaptación al medio ambiente de las distintas razas ovinas pudo haber ejercido su influencia en diferencias anatomo-fisiológicas capaces de permitir una capacidad de selección diferente sobre la cubierta vegetal.

Por otra parte, la evolución de la cubierta vegetal está condicionada a la acción de corte o utilización de la misma y por tanto, diferencias en la selección de los animales sobre el pasto pueden condicionar la evolución de la misma (Hepp, 1989).

Por todo ello, en este trabajo se pretenden conocer las diferencias en cuanto a la intensidad de selección del pasto de los dos genotipos ovinos más importantes de la región Castellano-Leonesa (raza Churra y raza Merina) en condiciones de pastoreo de montaña, y cómo esta intensidad de selección puede evolucionar a lo largo de la estación de pastoreo.

\section{Material y métodos}

El experimento se realizó en el parque de San Isidro (Isoba) en la provincia de León, a $1540 \mathrm{~m}$ de altitud, $43,03^{\circ}$ de latitud Norte y $01,41^{\circ} \mathrm{de}$ 
longitud Oeste. El clima de la zona es continental húmedo, la evolución de la pluviosidad y temperatura a lo largo del año se presentan en la figura 1 , indicando la media de los valores mensuales de los años 1960-1989, asi como los datos correspondientes al periodo de estudio (Junio-Septiembre. 1990).

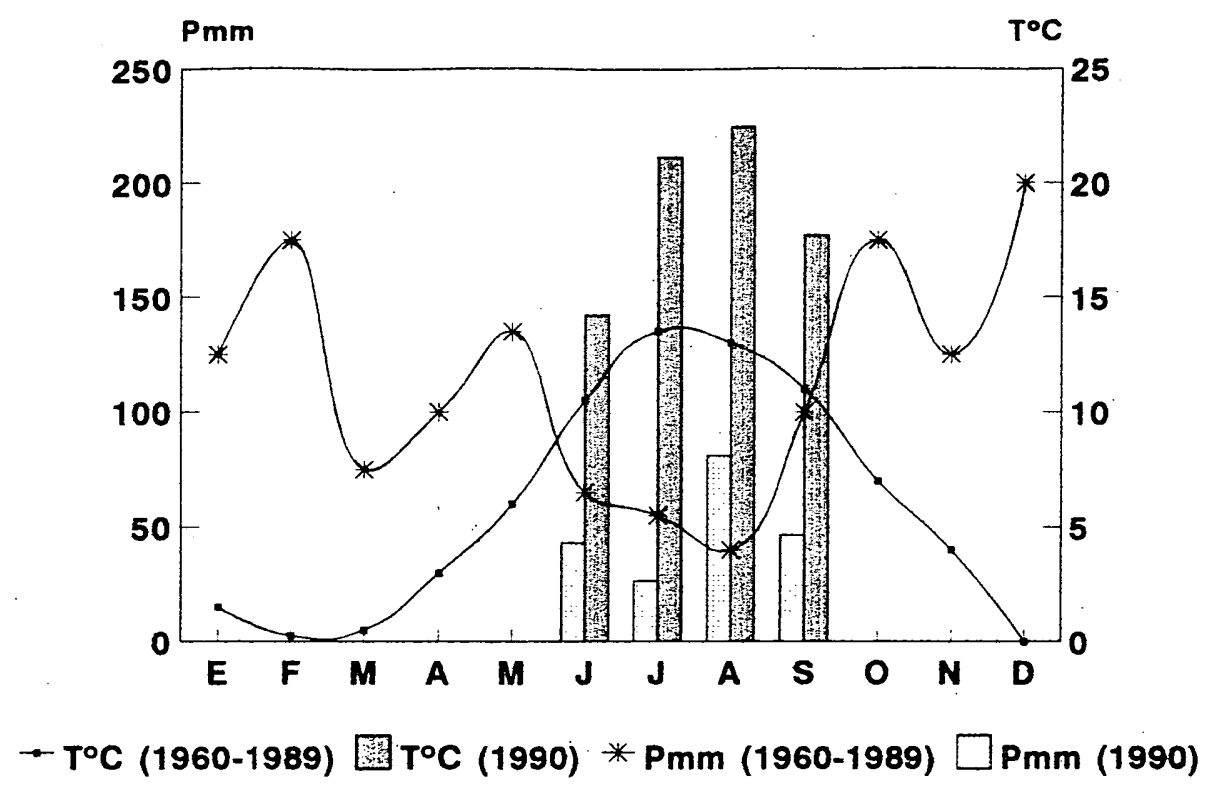

Figura 1. Variaciones climáticas anuales. Media de temperatura $\left(\mathrm{T}^{\circ} \mathrm{C}\right)$ y pluviometria en $\mathrm{mm}(\mathrm{Pmm})$ de los años 1960 a 1989 y del verano de 1990. (Yearly climatic variations. Average of temperature $\left(T^{\circ} \mathrm{C}\right)$ and precipitations in $\mathrm{mm}(\mathrm{Pmm})$ between 1960 and 1989 and the summer of 1990).

La cubierta vegetal de la parcela en estudio es mayoritariamente de "Nardus-Festuca", con una cobertura de 47,40 especies por $\mathrm{m}^{2} \mathrm{y}$ fueron identificadas 20 especies diferentes, todas ellas herbáceas, con una distribución de: $30 \%$ de Gramíneas, $5 \%$ de Leguminosas y $65 \%$ de Otras. La superficie de muestreo tenía una extensión aproximada de $300 \mathrm{~m}^{2}$, estando cercada con una malla metálica de $1,10 \mathrm{~m}$ de altura, permitiendo únicamente el pastoreo al ganado ovino.

Los muestreos fueron realizados durante la última semana de los meses de junio, julio, agosto y septiembre de 1990. Se utilizaron 8 machos adultos castrados ( 4 de raza Churra y 4 de raza Merina) provistos de una fístula esofágica, siguiendo la metodología para su realización y mantenimiento descrita por (Van Dyne \& Torell, 1964). El muestreo se realizó durante 2 dias consecutivos a primera hora de la mañana, habiendo sido encerrados los 
animales durante la noche. En el momento de iniciar el muestreo se retiraba de la fístula esofágica la placa y se colocaba una bolsa de plástico alrededor del cuello de cada animal para la recogida del pasto ingerido (extrusa).

El pasto disponible era evaluado al inicio de cada periodo de muestreo, cortándose al nivel del suelo y al azar 2 cuadrados de $0,25 \mathrm{~m}^{2}$. Las muestras recogidas, tanto de extrusa como de pasto, fueron conservadas a $20^{\circ} \mathrm{C}$, hasta la finalización del experimento, siendo liofilizadas posteriormente las muestras de extrusa para su separación manual en hojas, tallos y restos (considerando como tales el material no identificable, el cual se separaba por cribado). La separación de los diferentes componentes de la extrusa fue realizada independientemente para cada animal y cada día de muestreo. Las muestras de pasto se secaron en una estufa de aire asistido a una temperatura de $60^{\circ} \mathrm{C}$ durante 72 horas. La intensidad de selección ejercida por los animales se estimó de acuerdo con la fórmula:

$\mathrm{SI}=\mathrm{D} / \mathrm{F}$

Donde: $\mathrm{SI}$ = Indice de selección

$D=$ proporción de hojas en la extrusa

$\mathrm{F}=$ proporción de hojas en el pasto disponible,

considerando que los valores iguales a 1 suponen que el animal no ejerce ninguna presión de selección sobre el pasto y que los aumentos en el valor SI por encima de 1 indican una mayor intensidad de selección del pasto por el animal.

Los resultados de proporción de hojas en las extrusas y de índice de selección fueron sometidos a un análisis de varianza factorial según el modelo:

$$
u=x+A i+B j+A B i j+E
$$

Donde Ai representa el efecto debido al periodo, $\mathrm{Bj}$ el efecto debido al genotipo y ABij la interacción de ambos.

\section{Resultados}

Como puede observarse en la tabla 1. existe una distribución muy variable en la oferta de materia seca a lo largo del periodo de pastoreo estival, con valores similares en la disponibilidad en los meses de junio y agosto y un descenso, con valores también similares en la disponibilidad en los meses de julio y septiembre.

Para la proporción de hojas en la materia seca disponible se encuentran valores similares en los meses de julio, agosto y septiembre y un valor superior en el inicio de la estación de pastoreo. 
PIRINEOS 143-144

TABLA 1.

Oferta y proporción de hojas en el pasto.

\begin{tabular}{lcccc}
\hline & junio & julio & agosto & septiembre \\
Oferta (kg MS/Ha) & 1890 & 1209 & 1723 & 1206 \\
Hojas (\% MS) & 63,45 & 49,00 & 47,92 & 49,67 \\
\hline
\end{tabular}

Los valores de la proporción de hojas en la extrusa seleccionada por los animales se expresan en función de la suma de la materia seca de las hojas más los tallos y se indican en la tabla 2.

Se pueden observar diferencias estadísticamente significativas en la proporción de hojas en las extrusas de los distintos periodos, con un valor significativamente menor $(P<0,001)$ en el periodo de finales de julio, no encontrándose diferencias entre los otros tres periodos.

No se encontraron diferencias estadísticamente significativas $(P>0,05)$ entre los valores de la proporción de hojas de las extrusas de los animales de raza Churra y de raza Merina. Sin embargo, la interacción del efecto de raza $\times$ periodo fue significativo $(P<0,05)$, encontrando que las diferencias entre periodos en la proporción de hojas en las extrusas únicamente se presentan en los animales de raza Merina, no existiendo diferencias significativas entre los valores correspondientes a los animales de raza Churra.

Para los valores del Indice de selección indicados en la tabla 3 , se encontraron diferencias estadisticamente significativas $(P<0,001)$ por efecto

TABLA 2

Proporción de hojas en las extrusas (\% de la materia seca).

\begin{tabular}{lccc}
\hline & Churra & Merina & Media \\
junio & 89,93 & 89,41 & 89,67 \\
Julio & 84,84 & 68,29 & 77,75 \\
agosto & 90,62 & 92,93 & 91,67 \\
septiembre & 88,38 & 91,65 & 90,20 \\
Media & 80,28 & 85,33 & 86,87 \\
& & & \\
& & Factor de variación & \\
Error standard & Periodo & Genotipo & Periodo X Genotipo \\
Nivel de & 2,42 & $\mathbf{1 , 6 8}$ & 3,44 \\
significación (P<) & 0,0002 & 0,1336 & 0,0136 \\
\hline
\end{tabular}

48 
del periodo, con un menor valor en los meses de junio y julio frente a los periodos de agosto y septiembre, entre los cuales no se encontraron diferencias significativas; no pudiendo demostrar la existencia de diferencias estadisticamente significativas $(P>0,05)$ entre los valores de las dos razas estudiadas.

El hecho de que la interacción del periodo $x$ raza fue significativa indica que mientras para la raza Merina se mantiene el mismo patrón ya indicado en el efecto del periodo, en la raza Churra las diferencias en el Indice de selección se manifiestan con un valor significativamente menor en el periodo de junio frente a los valores de los otros 3 meses, entre los cuales no se encontraron diferencias.

TABLA 3

Indices de selección del pasto (\% hojas en la extrusa / \% hojas en el pasto).

\begin{tabular}{lccc}
\hline & Churra & Merina & Periodo x Genotipo \\
Junio & 1,42 & 1,41 & 1,41 \\
Julio & 1,73 & 1,39 & 1,59 \\
agosto & 1,89 & 1,94 & 1,91 \\
septiembre & 1,78 & 1,85 & 1,82 \\
Media & 1,68 & 1,61 & 1,64 \\
& & & \\
& & Factor de variación & \\
& Periodo & Genotipo & Periodo \\
Error standard & 0,0486 & 0,0338 & 0,0692 \\
Nivel de \\
significación $(P<)$
\end{tabular}

\section{Discusión}

Las variaciones mensuales encontradas en la oferta de materia seca a lo largo del verano pueden ser explicadas teniendo en cuenta los datos climatológicos. La mayor pluviosidad a finales del mes de agosto produce un estímulo del crecimiento vegetal, disminuyendo la disponibilidad de pasto en el mes de julio como consecuencia de la sequía estival y en el mes de septiembre por las menores temperaturas.

Diversos autores (Penning et al., 1991; Poppi et al., 1980) han puesto de manifiesto que las variaciones en el valor nutritivo del pasto pueden ser explicadas en función de los cambios en diversidad botánica y, dentro de un 
mismo pasto, por los cambios en la relación entre hojas y tallos asícomo por las variaciones en la proporción entre el material vegetal vivo y muerto en el pasto.

El hecho de no encontrar diferencias en la proporción de hojas del pasto entre los meses de julio, agosto y septiembre a pesar de las diferencias en la materia seca disponible, puede ser debido al efecto del pastoreo durante los periodos previos lo cual permitirla una constancia en la distribución del horizonte foliar (Grant \& Hodgson, 1986).

El menor valor de la proporción de hojas en las extrusas en el periodo de muestreo de julio, como consecuencia del descenso en este valor en los animales de raza Merina, coincidiría con los resultados obtenidos por Black \& Kenny, 1984, Hodgson et al., 1991 y Penning et al., 1991, quienes indican que al envejecer el pasto y disminuir la oferta del mismo, la intensidad de selección que los animales pueden ejercer sobre el mismo aumenta. Es necesario tener en cuenta que una menor oferta de pasto, por debajo de ciertos límites, está unida a una meńor proporción de tallos y envejecimiento del pasto, lo cual limita la posibilidad de selección en algunos genotipos ovinos.

Sin embargo, el hecho de no encontrar diferencias en la proporción de hojas de la dieta, como indicativo del valor nutritivo de la misma, en los animales de raza Churra y si en los de raza Merina, pone de manifiesto que la adaptación de los animales a lo largo del tiempo a distintos sistemas de manejo y alimentación determina variacionesen su comportamiento ingestivo en pastoreo (Hanley, 1982; Provenza \& Balph. 1987).

Los valores del índice de selección obtenido en la raza Merina en los periodos de junio y julio pueden ser explicados, el primero por la mayor oferta en este momento inicial y en julio por la disminución en la oferta como consecuencia de la sequia. El mayor valor de los indices de selección obtenidos en los periodos de agosto y septiembre serían consecuencia del crecimiento vegetal obtenido en agosto y el mantenimiento del mismo, aunque más ralentizado en el mes de septiembre.

El comportamiento ingestivo de los animales en pastoreo depende de las características de la cubierta vegetal y del tipo de animal. Los resultados obtenidos en cuanto a la relación oferta-intensidad de selección sobre el pasto coinciden con los obtenidos por Milne et al. (1978-1982), en el sentido de ejercer una mayor presión de selección sobre los componentes minoritarios del pasto 0 , tomando un componente del pasto como referencia, cuando la proporción de este componente disminuye.

A la vista de los resultados de los índices de selección que los animales ejercen sobre el pasto, debemos tener en cuenta que la ingestión de los animales en pastoreo es el resultado del producto del tamaño de cada bocado por el ritmo de ingestión y por el tiempo que está pastando (Forbes, 1988; Hodgson, 1985). De estos tres componentes, parece ser que es el tamaño de cada bocado el que ejerce una influencia más directa sobre la ingestión y en relación con la intensidad de selección, al aumentar ésta, el 
tamaño del bocado disminuye, ocurriendo de manera similar en la relación con el ritmo de ingestión (Hodgson, 1985; Penning, 1986). De manera que el mayor valor nutritivo de la dieta en el caso de los animales de raza Churra podría verse compensado en el caso de la raza Merina por una mayor ingestión total.

Sin embargo, es necesario tener en cuenta la distribución del material vegetal en el horizonte vegetal para diferenciar si la selección ejercida por los animales es un proceso activo o únicamente es consecuencia de la profundidad del horizonte foliar sobre el que realmente actúa el animal en pastoreo.

Tradicionalmente, la raza Churra ha sido explotada en la zona de la meseta castellano-leonesa, viéndose obligada a pastar sobre zonas arbustivas, de rastrojos, etc., donde la toma de alimentos debe de ser mucho más precisa. Por otra parte, el ganado merino ha sido explotado tradicionalmente, en un régimen de trashumancia o transterminancia sobre pastos, en general, de buena calidad y, por lo tanto, la necesidad de selección es mucho menos necesaria para la adquisición de nutrientes.

Las variaciones encontradas en la intensidad de selección de los animales de raza Churra y Merina en pastoreo deben de ser explicadas en relación con las modificaciones en las características anatómicas y fisiológicas de su aparato digestivo, en el sentido de que una menor intensidad de selección debería ir acompañada de una boca más ancha y de mayores dimensiones asi como por un mayor desarrollo del aparto digestivo y, por lo tanto, mayor capacidad de utilización de forrajes de baja calidad (Gordon \& Illius, 1988; Illius \& Gordon, 1987).

Desde un punto de vista de mejora y conservación del medio natural, la alternativa entre utilizar una y otra raza o ambas conjuntamente, a partir de estos resultados iniciales que deben de ser comprobados con mayor información, debe tener en cuenta que ante un pasto en el inicio de su degradación por abandono en su utilización, el ganado Merino podría hacer una función de limpieza más intensa y más eficiente.

El pastoreo mixto de Churras y Merinas, a pesar de la tradición popular, debe ser estudiado para conocer la influencia que pudiera tener sobre la evolución en la diversidad botánica y de manera especial en condiciones de pastoreo en zonas arbustivas, por la posible mayor facilidad de la raza Churra para cubrir sus necesidades a partir de las partes de mayor valor nutritivo.

Agradecimientos. Este trabajo forma parte del proyecto titulado "Utilización de pastos de montaña por el ganado ovino", subvencionado por la Excma. Diputación de León y ha sido realizado dentro del convenio específico de colaboración entre la Excma. Diputación Provincial de León y la Estación Agrícola Experimental del CSIC. 


\section{Referencias}

Armstrong, R.H. \& Hodgson, J. (1986): Grazing behaviour and herbage intake in cattle and sheep grazing indigenous hill plant communities. En: Grazing research at northern latitudes (ed. O. Gudmundson). pp. 211-218, Plenum Press, London.

Belovsky, G.E. (1986): Optimal foraging and community structure: implications for a guild of generalist herbivores. Oecologia, 70: 35-52.

Black, J.L. \& Kenny, P.A. (1984): Factors affectring diet selection by sheep. II. Height and density of pasture. Australian Journal of Agricultural Research, 35: 565-578.

Forbes, T.D.A. (1988): Researching the plant-animal interface: the investigation of ingestive behavior in grazing animals. Journal of Animal Science, 66: 2369-2379.

Gordon, I.J. \& lason G.R. (1989): The foraging strategy of rumiants: its significance to vegetation utilization and management. Macaulay Land Use Research Institute, Annual Report 1988, pp. 34-41, MLURI, Edinburgh.

Gordon, I.J. \& Illius, A.W. (1988): Incisor arcade structure and diet selection in rumiants. Functional Ecology, 2: 15-22.

Grant, S.A. \& Hodgson, J. (1986): Grazing effects on species balance and herbage production in indigenous plant communities. En: Grazing research at northern latitudes (ed. O. Gudmundson), pp. 69-77, Plenum Press, London.

Hanley, T.A. (1982): The nutritional basis for food selection by ungulates. Journal of Range Mangement, 35: 146-151.

Hepp, C. (1989): Interactions between sward conditions and the intake and grazing behaviour of sheep in the autumn. M. Phill. Thesis, University of Edinburgh.

Hodgson, J. (1985): The control of herbage intake in the grazing ruminant. Proceedings of the Nutrition Society, 44: 339-346.

Hodgson, J. (1989): Management of grazing systems. Proceedings of the New Zealand Grassland Association, 50: 117-122.

Hodgson, J., Forbes, T.D.A., Armstrong, R.H., Beattie, M.N. \& Hunter, E.A. (1991): Comparative studies of the ingestive behaviour and herbage intake of sheep and cattle grazing indigenous hill plant communities. Journal of Applied Ecology, 28: 205-227.

Illius, A.W. (1986): Foraging behaviour and diet selection. En: Gudmundson, 0 . (ed.) Grazing research at northern latitudes, pp. 227-236: Plenum in association with NATO Scientific Affairs Division, London.

Illius, A.W. \& Gordon, l.J. (1987): The allometry of food intake in grazing ruminants. Journal of Animal Ecology, 56: 989-999.

Malacheck, J.C. \& Balph, D.F. (1987): Diet selection by grazing and browsing livestock En: Hacker, J.B., Ternouth, J.H. (ed.). The nutrition of Herbivores, pp. 121-132, Academic Press, Sydney.

Milne, J.A., Hodgson, J., Thompson, R., Souter, W.G. \& Barthram, G.T. (1982): The diet ingested by sheep grazing swards differing in white clover and perennial ryegrass content. Grass and Forage Science, 37: 209-218.

Milne, J.A., Macrae, J.C., Spence, A. \& Wilson, S. (1978): A comparison of the coluntary intake and digestion of forages at different times of the year by the sheep and red deer (Cervus elaphus). British Journal of Nutrition, 40: 347-357.

Penning. P.D. (1986): Some effects of sward contitions on grazing behaviour and intake by sheep. En: Gudmundson, O. (ed.) Grazing research at northern latitudes. pp. 219226, Plenum in Association with NATO Scientific Affairs Division, London. 
ESTUDIO DE LAS COMUNIDADES DE INTERES PASCICOLA

Penning, P.D. Parsons, A.J., Orr, R.J. \& Treacher, T.T. (1991): Intake and behaviour responses by sheep to changes in sward characteristics under continuous stocking. Grass and Forage Science, 46: 15-28.

Poppi, D.P., Minson, D.J. \& Ternouth, J.H. (1980): Studies of cattle and sheep eating leaf and stem fractions of grasses. I. The voluntary food intake, digestibility and retention time in the reticulum-rumen. Australian Journal of Agricultural Research, 32: 99-108.

Provenza, F.D. \& Balph, D.F. (1987): Diet learning by domestic ruminants: theory, evidence and practical implications. Applied Animal Behaviour Science, 18: 211 232.

Van Dyne, G.M. \& Torell, D.T. (1964): Development and use of the esophageal fistula. Journal of Range Management, 17: 7-19.

Weston, R.H. \& Poppi, D.P. (1987): Comparative aspects of food intake. En: Hacker, J.B., Ternouth, J.H. (ed.) The nutrition of Herbivores, pp. 133-161, Academic Press, Sydney. 